\title{
Comparison of parametric and non-parametric methods for bladed disks with random geometric mistuning
}

\author{
Maxime Koebele ${ }^{1, *}$ and Carsten Proppe ${ }^{1}$ \\ ${ }^{1}$ Karlsruhe Institue of Technology (KIT), Institute of Engineering Mechanics, Kaiserstr. 10, 76131 Karlsruhe
}

This paper presents different strategies to quantify the influence of random geometric mistuning on the dynamic behavior of bladed disk. These strategies are compared for different correlation lengths of the random field describing the blade geometry. The results allow to determine the kind of random geometric mistuning for which these strategies are appropriate.

\section{Introduction}

Mistuning of a bladed disk denotes the loss of cyclic symmetry due to material and geometric imperfections arising along the blades during the manufacturing process. Mistuning may lead to significant increase in forced-response vibration amplitudes for certain blades. Several methods were developped in the last two decades to quantify this phenomenon. Today, an emerging topic is to implement mistuning in a realistic way, for example by considering random geometric mistuning.

\section{Implementation of geometric uncertainites}

Random geometric mistuning can be introduced in the FEM model of a bladed disk via random variations of blade surfaces in normal direction, which can be represented by a mean-zero Gaussian random field with an exponential covariance function :

$$
c(\mathbf{x})=\sigma \exp \left(-\frac{\|\mathbf{x}\|_{2}^{2}}{l_{c}^{2}}\right)
$$

where the parameter $\sigma$ (resp. $l_{c}$ ) allows to specify a tolerance domain (resp. a correlation length). The random field is then spatially discretized with respect to the FEM mesh so that the geometry of each blade $i$ depends on a random input vector $\boldsymbol{\xi}_{i}$.

\section{Parametric and non parametric reduced-order models}

If Monte-Carlo simulations are then performed, two difficulties occur. First, the computational cost due to the FEM analysis necessitates the use of reduced-order models. Secondly, the computation of structural matrices for each realization of the random input vectors $\boldsymbol{\xi}_{i}$ leads to unreasonable computational cost. The following methods allow to overcome these difficulties.

\subsection{Parametric method using random material parameters (SNM)}

Here, it is assumed that random geometric mistuning can be approximated by considering only the Young's modulus and the density of the blades as random parameters without geometric modifications of the mesh. Probabilistic models for these two parameters are defined such that equivalent deviations in the norm of the mass and stiffness matrix are obtained. Structural matrices can be here easily and quickly computed and then reduced using a subset of nominal modes (SNM) as shown in [1].

\subsection{Non parametric method using random reduced matrices (SNM NP)}

Another idea is to randomly generate the reduced matices using the maximum entropy principle regardless of the blade geometry as shown in [2]. Thus, the reduced matrices are no longer functions of the random input parameters. Only the dispersion parameters controlling their random generation are estimated depending on the geometric tolerances. Furthermore, a subset of nominal modes can also be used here as reduction basis.

\subsection{Parametric method using a subset of stochastic modes (SSM)}

The last possibility is to use a subset of stochastic modes depending on the random input vectors $\boldsymbol{\xi}_{i}$ to build a reduction basis. These stochastic modes are the solution to a random eigenvalue problem corresponding to a bladed disk with all blades having the same random geometry. Using the modal and orthogonal properties of these modes, the reduced matrices can be computed without even having an analytical formulation of the structural matrices of the full system as shown in [3].

\footnotetext{
* Corresponding author: e-mail maxime.koebele@ kit.edu, phone +49 721608 42660, fax +4972160846070
} 


\section{Numerical results}

The three methods are compared for an academic disk with 30 blades, random geometric uncertainties with a small correlation length $\left(l_{c}=10 \mathrm{~mm}\right)$ and with large correlation length $\left(l_{c}=190 \mathrm{~mm}\right)$ are considered. For both cases, the parameter $\sigma$ is fixed in such a way that the blade surfaces are contained in a tolerance domain of $\pm 0.1 \mathrm{~mm}$ with a confidence of $99 \%$. Resonance frequencies are then computed using Monte-Carlo simulations for 10000 mistuned bladed disks.

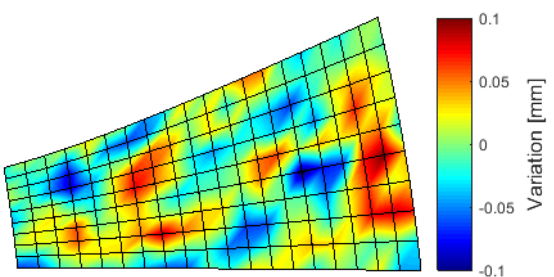

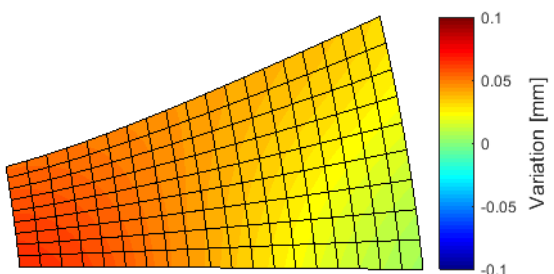

Fig. 2: Variation of blade surface for one realization in case of large correlation length $\left(l_{c}=190 \mathrm{~mm}\right)$

Fig. 1: Variation of blade surface for one realization in case of small correlation length $\left(l_{c}=10 \mathrm{~mm}\right)$

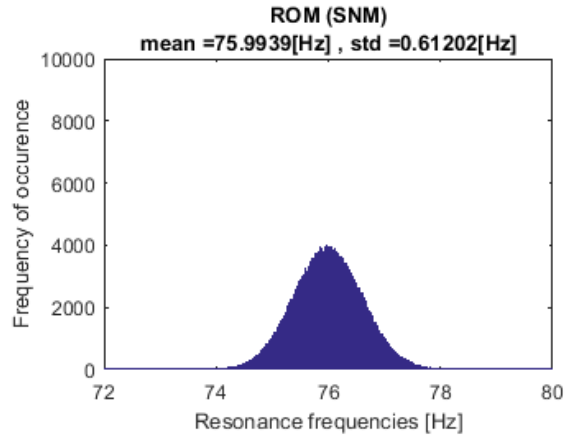

Fig. 3: Histogram of resonance frequencies / small correlation length / SNM method

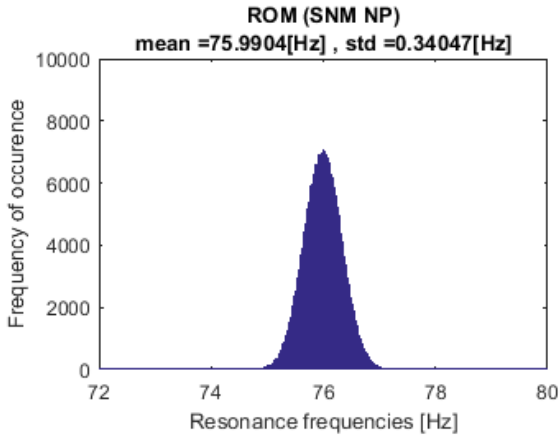

Fig. 4: Histogram of resonance frequencies / small correlation length / SNM NP method

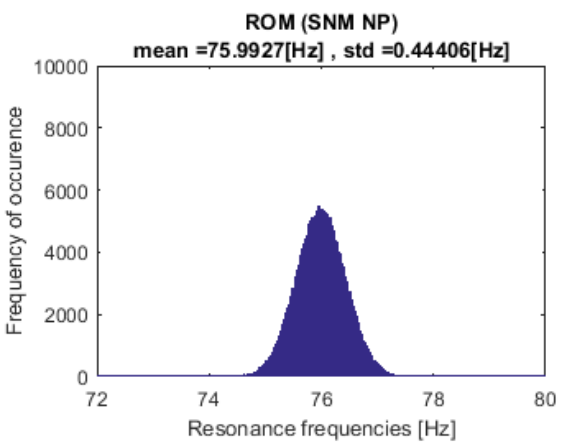

Fig. 7: Histogram of resonance frequencies / large correlation length / SNM NP method

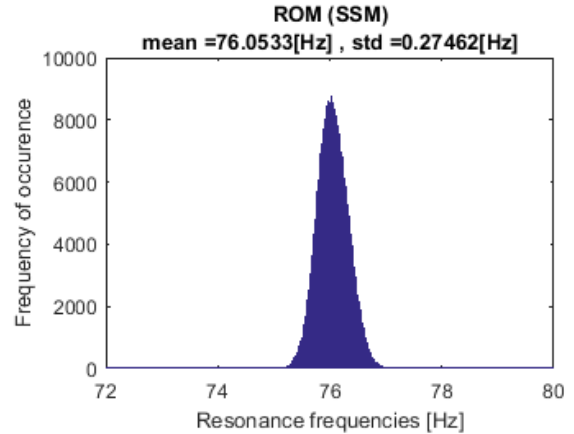

Fig. 5: Histogram of resonance frequencies / small correlation length / SSM method

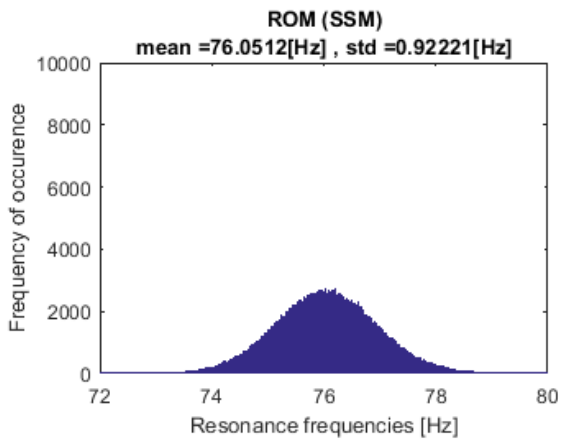

Fig. 8: Histogram of resonance frequencies / large correlation length / SSM method SNM method

Since the SSM method is the only one which does not use a simplifed mistuning implementation, it is taken as a reference. The comparison indicates that the SNM method with random material parameters seems to be suitable only in case of large correlation length (uniform deviation) and the SNM NP method with random reduced matrices seems to be suitable only in case of small correlation length (disordered deviation).

\section{References}

[1] M.-T. Yang and J.H. Griffin. A reduced-order model of mistuning using a subset nominal system modes. Journal of Engineering for Gas Turbines and Power 123(4), 893-900 (2001).

[2] E. Capiez-Lernout, C. Soize, J.-P. Lombard, C. Dupont and E. Seinturier . Blade manufacturing tolerances definition for a mistuned industrial bladed disk. Journal of Engineering for Gas Turbines and Power 127(3), 621-628 (2005).

[3] M. Koebelé-Cousquer and C. Proppe. Geometric uncertainties in finite element models of mistuned bladed disks. Proceedings in Applied Mathematics and Mechanics. 16, 671-672 (2016). 


\section{Repository KITopen}

Dies ist ein Postprint/begutachtetes Manuskript.

Empfohlene Zitierung:

Koebele, M.; Proppe, C.

Comparison of parametric and non-parametric methods for bladed disks with random geometric mistuning.

2017. Proceedings in applied mathematics and mechanics, 17.

doi:10.5445/IR/1000090314

Zitierung der Originalveröffentlichung:

Koebele, M.; Proppe, C.

Comparison of parametric and non-parametric methods for bladed disks with random geometric mistuning.

2017. Proceedings in applied mathematics and mechanics, 17 (1, SI), 723-724.

doi:10.1002/pamm.201710330 